\title{
Rubber band ligation versus haemorrhoidectomy for the treatment of grade II-III haemorrhoids: a systematic review and meta-analysis of randomised controlled trials
}

\author{
L. Dekker ${ }^{1,2}$-I. J. M. Han-Geurts ${ }^{2} \cdot$ H. D. Rørvik ${ }^{3,4} \cdot$ S. van Dieren ${ }^{1} \cdot$ W. A. Bemelman ${ }^{1}$
}

Received: 10 September 2020 / Accepted: 15 February 2021 / Published online: 8 March 2021

(c) The Author(s) 2021

\begin{abstract}
Background The aim of this study was to review clinical outcome of haemorrhoidectomy and rubber band ligation in grade II-III haemorrhoids.

Methods A systematic review was conducted. Medline, Embase, Cochrane Library, Clinicaltrials.gov, and the WHO International Trial Registry Platform were searched, from inception until May 2018, to identify randomised clinical trials comparing rubber band ligation with haemorrhoidectomy for grade II-III haemorrhoids. The primary outcome was control of symptoms. Secondary outcomes included postoperative pain, postoperative complications, anal continence, patient satisfaction, quality of life and healthcare costs were assessed. Preferred Reporting Items for Systematic Reviews and Meta-Analyses (PRISMA) guidelines were followed.

Results Three hundred and twenty-four studies were identified. Eight trials met the inclusion criteria. All trials were of moderate methodological quality. Outcome measures were diverse and not clearly defined. Control of symptoms was better following haemorrhoidectomy. Patients had less pain after rubber band ligation. There were more complications (bleeding, urinary retention, anal incontinence/stenosis) in the haemorrhoidectomy group. Patient satisfaction was equal in both groups. There were no data on quality of life and healthcare costs except that in one study patients resumed work more early after rubber band ligation.

Conclusions Haemorrhoidectomy seems to provide better symptom control but at the cost of more pain and complications. However, due to the poor quality of the studies analysed/it is not possible to determine which of the two procedures provides the best treatment for grade II-III haemorrhoids. Further studies focusing on clearly defined outcome measurements taking patients perspective and economic impact into consideration are required.
\end{abstract}

Keywords Haemorrhoids $\cdot$ Rubber band ligation $\cdot$ Haemorrhoidectomy $\cdot$ Complications $\cdot$ Reintervention

L. Dekker, I.J.M. Han-Geurts, H. Rørvik, S. van Dieren and W.A. Bemelman contributed equally in writing.

S. van Dieren was responsible for the statistical analysis.

L. Dekker

1.dekker22@amsterdamumc.nl

1 Department of Surgery, Amsterdam University Medical Center, Location AMC, Meibergdreef 9, 1105 AZ Amsterdam, The Netherlands

2 Department of Surgery, Proctos Clinic, Bilthoven, The Netherlands

3 Department of Surgery, Holbæk Hospital, Holbæk, Denmark

4 Department of Acute and Digestive Surgery, Haukeland University Hospital, Bergen, Norway

\section{Introduction}

Haemorrhoids are one of the most common proctological disorders with an incidence of about $9 / 1000$ patients per year in the Netherlands [1] and a prevalence up to $39 \%$ in the general population [2]. Treatment consists initially of conservative measures such as lifestyle advice, diet and toilet behaviour. In addition, there are various surgical options, Haemorrhoidectomy is considered the gold standard and this was recently confirmed in a British trial and systematic review [3, 4]. The most common minimally invasive procedure is rubber band ligation (RBL). Other minimally invasive procedures are sclerotherapy and laser treatment. These treatments are usually reserved for grade I and II haemorrhoids, although RBL is also used for grade 
III $[5,6]$. Grade III and IV haemorrhoids can be treated with open haemorrhoidectomy, semi-closed haemorrhoidectomy, and stapled haemorrhoidectomy with possibly mucopexy or haemorrhoidal artery ligation (HAL).

Many studies and meta-analyses have been published on the subject of haemorrhoid treatment. All these studies focus on groups of comparable surgical procedures. It is common to distinguish between minimally invasive treatment for grade II and III diseases (sclerotherapy and RBL) and surgical procedures for grade III and IV haemorrhoids (haemorrhoidectomy and stapled haemorrhoidectomy). However, the criteria for selecting a minimally invasive treatment versus an operation are not always that evident. There is obviously an overlap in indication, as has become clear from several surveys amongst treating surgeons [7, 8]. There are few trials comparing the clinical outcome of the two most common treatments RBL and haemorrhoidectomy. A systematic review from 2005, updated in 2016, of 3 small heterogeneous trials concluded that RBL leads to a higher recurrence rate, but on the other hand less pain, fewer complications, and a less stressful experience for the patient $[9,10]$.

It remains unclear which of the two most common procedures is preferable as regards healthcare costs. There are hardly any studies investigating the cost effectiveness of the various treatments. Only 1 study compared costs of stapled haemorrhoidopexy with RBL in grade II haemorrhoids with results in favour of RBL [11]. A recent study from 2016 compared HAL with RBL, with HAL clearly entailing higher costs, even though the analysis includes the possibility of repeated RBL treatments [12]. Since haemorrhoidal disease is a benign condition, the main goal of treatment is the resolution of symptoms and improvement of patient wellbeing. It is, therefore, important to include patient-related outcomes when determining the best treatment.

The aim of this systematic review was to assess the literature on the clinical effectiveness (including patient-related outcomes) and cost effectiveness of RBL versus haemorrhoidectomy in patients with symptomatic grade II and III haemorrhoids.

\section{Materials and methods}

This systematic review was undertaken in accordance with the Preferred Reporting Items for Systematic Reviews and Meta-Analyses (PRISMA) guidelines [13]. To reduce the risk of bias, a study protocol was made at an early stage and stated precise eligibility criteria. The protocol was registered in PROSPERO (registration number CRD42018102000) [14].

\section{Search strategy}

A comprehensive literature search was carried out from inception until May 2018, using a combination of freetext terms and controlled vocabulary. Medline, Embase, Cochrane Library, Clinicaltrials.gov, and the WHO International Trial Registry Platform were searched to identify randomised clinical trials comparing RBL with haemorrhoidectomy. The references of the identified trials were also searched to find additional trials for inclusion. Only studies written in English were included. There were no restrictions on publication year or publication status.

\section{Search terms}

The following search terms were used:

("Hemorrhoids"[Mesh] OR hemorrhoid*[tiab] OR haemorrhoid*[tiab] OR piles[tiab]) AND ("Ligation"[Mesh] OR ligature*[tiab] OR ligation*[tiab] OR band*[tiab]) AND ("Surgical Procedures, Operative"[Mesh:NoExp] OR "Hemorrhoidectomy”[Mesh] OR "Diathermy”[Mesh] OR "Electrocoagulation" [Mesh] OR "Lasers"[Mesh] OR hemorroidectom*[tiab] OR haemorroidectom*[tiab] OR hemorrhoidectom*[tiab] OR haemorrhoidectom*[tiab] OR hemorrhoid excison*[tiab] OR haemorrhoid excison*[tiab] OR Milligan-Morgan[tiab] OR ferguson[tiab] OR ligasure[tiab] OR diathermy[tiab] OR harmonic scapel[tiab] OR electrocauter*[tiab] OR laser*[tiab] OR thermocoagulation[tiab]).

\section{Inclusion and exclusion criteria}

Randomised controlled trials (RCTs) comparing RBL to/ with haemorrhoidectomy in grade II-III haemorrhoids according to Goligher's classification were included in this systematic review. Only studies considering non-emergency procedures in adult patients and reporting of the required outcomes were included. Adult patients (18 years or older) were included and all techniques (open, semi-closed, and closed) or instruments (scissors, knife, diathermy, LigaSure, and harmonic scalpel) used for haemorrhoid excision were included. Non-randomised studies and studies not in English language were excluded.

\section{Quality assessment}

The methodological quality of the included studies was assessed using the following Cochrane Risk of Bias assessment tool: sequence generation, allocation concealment, blinding of participants and personnel, blinding of outcome assessors, incomplete outcome data, selective outcome 
reporting and other sources of bias [15]. Grading of Recommendations Assessment, Development, and Evaluation (GRADE) [16] was used to assess the quality (certainty) of evidence. It grades evidence as high, moderate, low or very low quality. Judgements included risk of bias, inconsistency, indirectness, imprecision and other considerations.

\section{Outcomes of interest}

The primary outcome was control of haemorrhoidal disease defined by need for retreatment within 1 year or by selfreported residual complaints. The secondary outcomes were postoperative pain, postoperative complications (bleeding requiring admission and/or reoperation, sepsis, anal stenosis, anal incontinence), anal continence (if measured by a validated patient-reported outcome measure), patient satisfaction, quality of life (if measured by a validated patientreported outcome measure), and health-costs. All complications reported (by studies) were added and reported individually.

\section{Data collection}

Literature search results were uploaded to Covidence Software. This is a Cochrane-supported software program that can import citations, screen titles, abstracts and full text. Data selection and extraction was conducted in accordance with Population, Interventions, Comparison, Outcome (PICOs). Identified trials were screened by two independent investigators. Titles, abstracts and full text were screened by both reviewers against inclusion and exclusion criteria. Trials that were excluded were documented with reasons for exclusion recorded. Efforts were made to contact trial investigators to resolve questions about eligibility or missing data but did not lead to additional data. The reviewers were not blinded to the journal titles or to study authors or institutions.

\section{Statistical analysis}

Binary data indicating number of patients with an event were analysed using a binomial model calculating risk ratio (RR) and 95\% confidence interval (CI). The estimates from individual RCTs were pooled using the random-effects model. Statistical heterogeneity was explored by $\chi^{2}$ test and expressed as $I^{2}$ and $p$ value (considered significant if $p<0.05$ ). The potential effect of predictors on the outcomes was investigated using a random-effects meta-regression model. Analyses were made using RevMan 5.3.5 (The Cochrane Collaboration) and RStudio.

\section{Results}

A total of 324 references were identified from the relevant electronic searches. Two duplicates were removed. Two hundred and ninety-five studies were excluded after screening titles and abstracts. Twenty-seven full-text studies were assessed for eligibility. Of these, 19 were excluded after fulltext review. Eight RCTs were identified and included in the analyses (Fig. 1) [17-24]. The risk of bias in the included trials is summarised in Fig. 2a, b. The overall methodological quality of these studies was determined to be moderate. The eight trials contained a total of 1208 patients with second- and third-degree haemorrhoids, who underwent RBL or haemorrhoidectomy (608 versus 600 , respectively). The characteristics of the studies are shown in Table 1.

\section{Recurrence and need for retreatment}

Recurrence was identified as outcome in 4 of the 8 trials. RBL led to more recurrence than haemorrhoidectomy (4 studies, 322 patients, random effects; RR 4.77 (95\% CI $2.60-8.76) ; p<0.001)$ as shown in Fig. 3). The index of heterogeneity between studies was assessed $\left(I^{2}\right)$ for a fixed effects model, and was low ( $0 \%)$. Recurrence of disease was established in different ways: need of reintervention [22]; diminishment of bleeding and prolapse [23] and recurrence of complaints $[18,20]$. GRADE evidence for recurrence within all included studies was very low (Table 2).

\section{Postoperative pain}

Patients experienced less post-procedural pain after RBL as demonstrated in Fig. 4 (7 studies, 1110 patients, RR 0.17 (95\% CI $0.11-0.28) ; p<0.001)$. Heterogeneity between studies was moderate $\left(I^{2}=76 \%, p<0.001\right)$. This statistical heterogeneity between the studies may be explained by variations in the method used to measure the postoperative pain or the moment it was scored. Often it was not even mentioned [17, 19, 20, 24]. Only Izadpanah et al. used the visual analog scale to measure the pain score which was in favor of RBL (5 versus 8) [21]. The GRADE-rated evaluation showed low quality of evidence due to downgrading on risk of bias, indirectness and imprecision.

\section{Postoperative bleeding}

Seven studies including 1110 patients and 84 events described postoperative bleeding as an outcome. This was less common following RBL [random effects; RR 0.31 (95\% CI $0.15-0.66) ; p=0.002$ ]. Heterogeneity between studies was moderate $\left(I^{2}=48 \%\right)$ (Fig. 5). None of the included 
Fig. 1 PRISMA flowchart of literature search

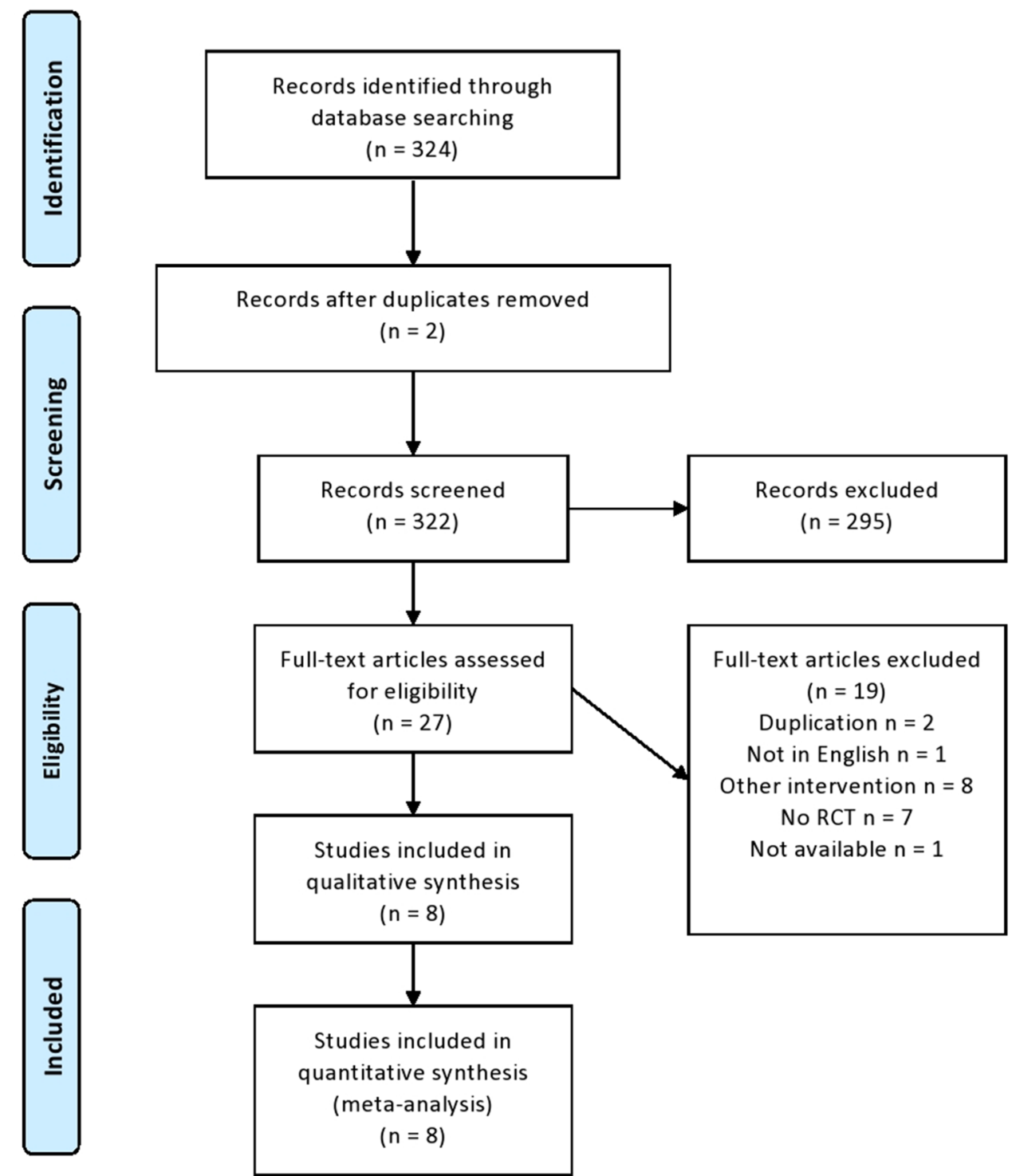

studies describes how this outcome was defined. Following haemorrhoidectomy, bleeding required reintervention in 15 patients [17, 18, 20, 22-24], Only Murie et al. reported that transfusion was the intervention used for their only patient with bleeding after haemorrhoidectomy. In the RBL arm, one patient needed readmission, no reintervention was described [22]. Quality of evidence was graded as very low for postoperative bleeding due to downgrading on risk of bias, indirectness and imprecision.

\section{Urinary retention}

Six studies reported data on urinary retention. All of them concluded that urinary retention requiring a urinary catheter is more common after haemorrhoidectomy than after RBL (6 studies, 1054 patients, random effects; RR 0.15 [95\% CI 0.09-0.25]; $p<0.001$ ) (Fig. 6). The rate of urinary retention was $0-4 \%$ after RBL versus $6.7-56 \%$ after haemorrhoidectomy. Due to downgrading on risk of bias and indirectness quality of evidence was assessed low.

\section{Anal continence and anal stenosis}

Anal incontinence was scored in 3 studies [17, 19, 21] and none of them found incontinence after RBL [236 patients, random effects; RR 0.16 [95\% CI 0.02-1.28] $p=0.080$ ) (Fig. 7)]. Ashghar et al. described incontinence in the haemorrhoidectomy group in, respectively, $5 \%$ and $7.7 \%$ of patients [19]. GRADE evidence for anal incontinence in all 3 studies was very low due to downgrading on risk of bias, indirectness and imprecision.

Five studies reported on anal stenosis (total of 942 patients, random effects; RR 0.11 [95\% CI 0.03-0.38] $p<0.001$ ) (Fig. 8). After haemorrhoidectomy, $1-8.3 \%$ of 
a

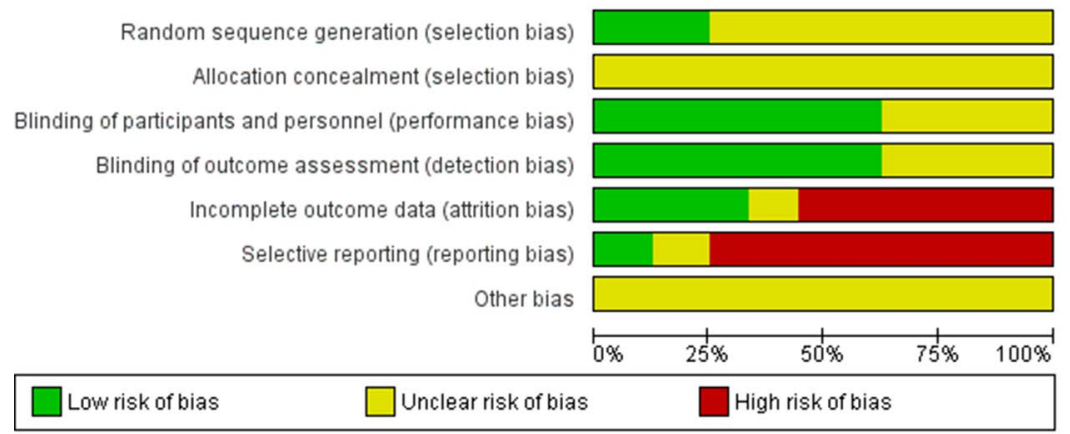

b

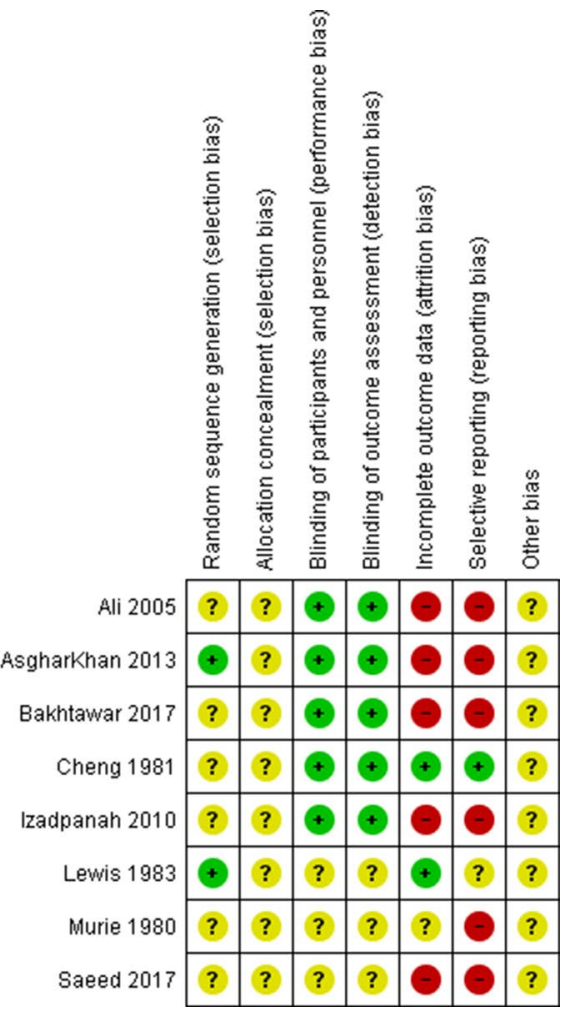

Fig. 2 a Summary of risk of bias across included studies. b Summary of risk of bias for each included study

patients developed anal stenosis. Stenosis following RBL only occurred in one patient [19]. Quality of evidence was stated to be low for this outcome.

\section{Patient satisfaction}

Murie et al. performed a patient assessment in which 93\% of patients undergoing haemorrhoidectomy had an excellent to moderately successful result versus $88 \%$ of patients undergoing rubber band ligation [22]. Ashgar et al. reported a patient satisfaction rate of $93 \%$ in the haemorrhoidectomy arm compared to $86 \%$ in the RBL arm [19]. This was due to the necessity of a repeat procedure in the RBL group. Regarding patient load, Saeed et al. reported a hospital stay of 2.5 days after haemorrhoidectomy versus 1 day after RBL [23]. Loss of working days following treatment was reported by Murie et al. favouring RBL (32 versus 3 days): this difference was statistically significant.

\section{Predictors for postoperative pain after RBL and haemorrhoidectomy}

The variable significantly associated with more post-procedural pain was age, which explained part of the heterogeneity. A meta-regression showed an age corrected RR of 0.23 for RBL compared to haemorrhoidectomy (95\% CI
$0.13-0.43, p<0.001)$. Only 5 trials were analysed, as Cheng et al. did not mention the standard deviation [17]. A metaregression on sex was not associated with postoperative pain and did not explain the heterogeneity $(p=0.560)$.

\section{Discussion}

The present study gives an update of the results of the two most commonly used strategies in treatment of grade II and III haemorrhoids. The results of this review suggest that haemorrhoidectomy is superior to RBL in reducing symptoms but is associated with more postoperative pain and adverse events. The review included only RCTs. Studies otherwise designed would result in an increase of bias. The overall quality of the included studies based on the Cochrane Collaboration's risk of bias tool was questionable. Incomplete outcome data (attrition bias) and selective reporting (reporting bias) were the major drawbacks. Furthermore, an important limitation was the lack of or poor definition of outcome measurements. The overall methodological quality of the included studies is moderate. Unfortunately, none of the included studies described the randomisation process and three of the eight studies compared more procedures than the two we were interested in. The included studies did not all use the same 


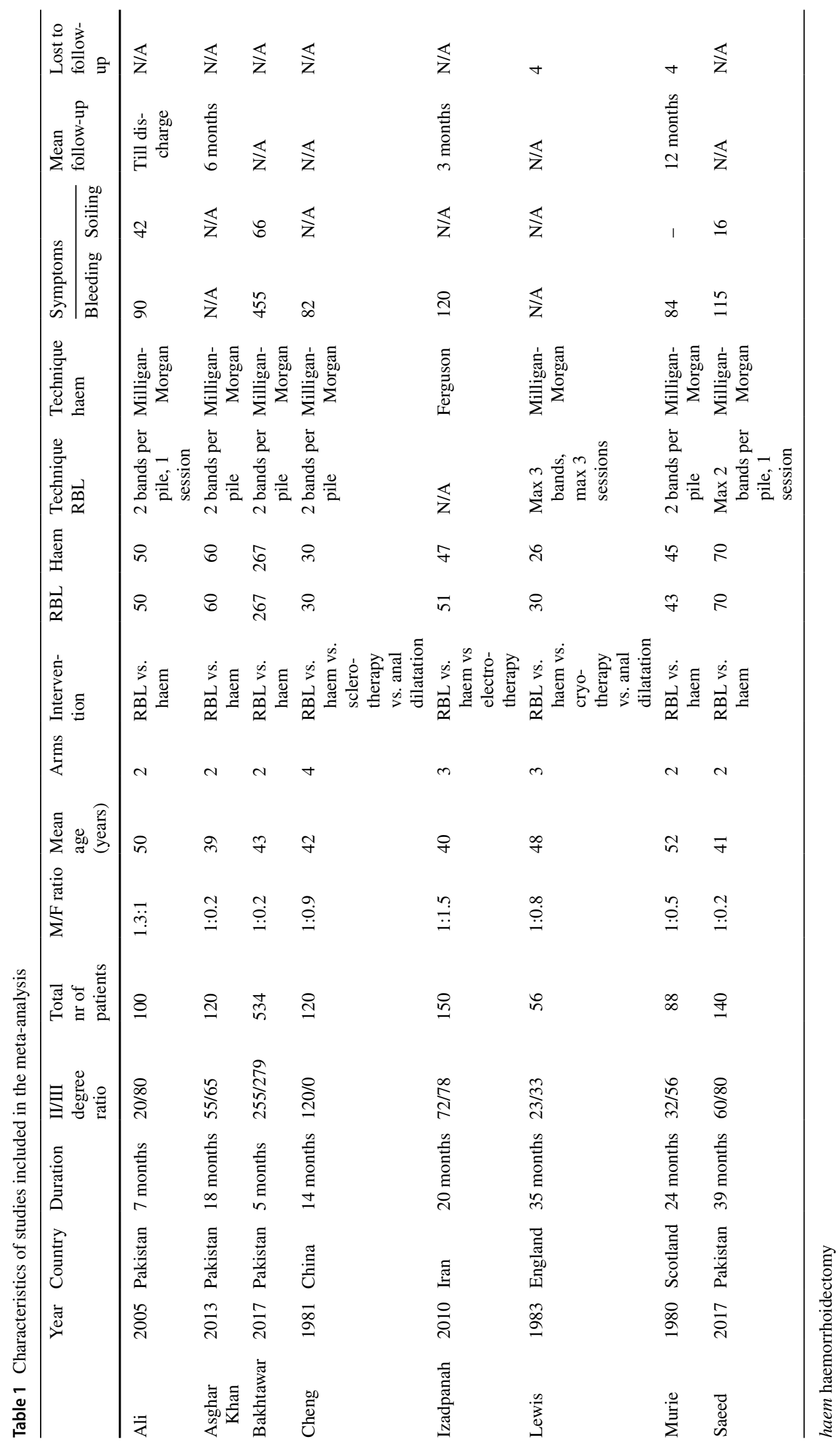




\begin{tabular}{|c|c|c|c|c|c|c|c|c|c|c|}
\hline \multirow[b]{2}{*}{ Study or Subgroup } & \multicolumn{2}{|c|}{ rubber band ligation } & \multicolumn{2}{|c|}{ hemorrhoidectomy } & \multirow{2}{*}{ Weight } & \multirow{2}{*}{$\begin{array}{c}\text { Risk Ratio } \\
\text { IV, } \text { Random, } 95 \% \mathrm{Cl}\end{array}$} & \multirow{2}{*}{\multicolumn{4}{|c|}{$\begin{array}{c}\text { Risk Ratio } \\
\text { IV, Random, } 95 \% \mathrm{Cl}\end{array}$}} \\
\hline & Events & Total & Events & Total & & & & & & \\
\hline AsgharKhan 2013 & 8 & 60 & 2 & 60 & $16.2 \%$ & $4.00[0.89,18.06]$ & & & & \\
\hline Cheng 1981 & 5 & 30 & 1 & 30 & $8.5 \%$ & $5.00[0.62,40.28]$ & & & & \\
\hline Lewis 1983 & 24 & 28 & 5 & 26 & $57.3 \%$ & $4.46[2.00,9.94]$ & & & & \\
\hline Murie 1980 & 13 & 43 & 2 & 45 & $18.1 \%$ & $6.80[1.63,28.39]$ & & & & \\
\hline Total $(95 \% \mathrm{Cl})$ & & 161 & & 161 & $100.0 \%$ & $4.77[2.60,8.76]$ & & & & \\
\hline Total events & 50 & & 10 & & & & & & & \\
\hline \multicolumn{7}{|c|}{$\begin{array}{l}\text { Heterogeneity: } \operatorname{Tau}^{2}=0.00 ; \mathrm{Chi}^{2}=0.32, \mathrm{df}=3(\mathrm{P}=0.96) ; \mathrm{I}^{2}=0 \% \\
\text { Test for overall effect: } Z=5.05(P<0.00001)\end{array}$} & 0.05 & 0.2 & 1 & 5 \\
\hline
\end{tabular}

Fig. 3 Recurrence rate. Relative risk values are shown with 95\% confidence intervals

techniques of haemorrhoidectomy and RBL applications and only 3 studies reported the length of follow-up which was, respectively, 3, 6 and 12 months [17, 21, 23]. The overall certainty of the evidence using the GRADE system was, therefore, low to very low (Table2). It should be noted that three studies $[18,22,23]$ are not of recent date but we still consider these relevant since the surgical procedures discussed have not changed since.

We defined control of haemorrhoidal disease by need for retreatment within 1 year or by self-reported residual complaints. Four studies report on effect of treatment and/or recurrence but a definition or follow-up is not given making results hard to interpret. Three studies only mention effect of treatment on bleeding, prolapse [18, 23] or pruritus [22] while other symptoms of haemorrhoidal disease are not mentioned. This makes it difficult to comment on efficacy of treatment. Other trials reporting on the outcomes of these procedures also demonstrate a lower recurrence rate after haemorrhoidectomy with the same limitations $[4,9,25]$. Besides, should repeated banding be considered as recurrence or part of the treatment? For re-banding, for instance, two or three sessions are common and patients may find this a more agreeable than one operation if the results are comparable in the long run. Except for 2 trials [17, 24], which reported performing 1 session of RBL, none of the included trials describes the exact number of RBL sessions.

Overall, postoperative complications were more common after haemorrhoidectomy. Postoperative bleeding and pain were mentioned in all studies and was more common following haemorrhoidectomy. However, none of the studies defined bleeding and only 1 used a visual analog scale to assess postoperative pain [21]. In addition, the timing of these outcome assessments was not specified in most studies. Pain after RBL has been analysed in other studies comparing RBL with more invasive procedures and was found to be less severe after RBL [12]. In a study by Watson et al. [26], 183 patients were asked to rate their pain on a scale of $1-5$ at different time points after RBL. The most severe pain was experienced at $4 \mathrm{~h}$ following RBL and after 1 week, $75 \%$ of the patients did not experience any pain at all. In the HubBle trial, pain was less after RBL compared to a surgical procedure (HAL) at 1 day (3.4 versus 4.6) and 1 week (1.6 versus
3.1) following the procedure [12]. After 3 and 6 weeks, pain scores were similar in both groups.

Urinary retention occurred far more often after haemorrhoidectomy. Rates of urinary retention are reported in the literature: $2-34 \%$ after haemorrhoidectomy and $0-0.4 \%$ after RBL [3, 27, 28]. The mechanism responsible for urinary retention is thought to be the triggering of a reflex leading to inhibition of the detrusor muscle. Pain and stretching of the anal canal may induce this reflex. The extent of surgical resection is related to the risk of developing urinary retention, probably due to more postoperative oedema and pain [29].

Anal incontinence following haemorrhoidectomy was reported in 3 studies [18, 20, 22] ranging from 0 to $7.7 \%$. Anal incontinence after RBL was not reported. This is in concordance with the recent literature [30]. However, none of the studies used a validated scoring system for anal incontinence. Other literature using the Vaizey or Cleveland incontinence score mention similar scores for RBL and HAL [12]. Anal stenosis was found in 1 patient after RBL and was not common after haemorrhoidectomy either (26/472) but this difference was significant. This stresses the importance of a careful surgical technique in performing haemorroidectomy which is sometimes is considered simple surgery.

Treatment patients complaining of haemorrhoids aims to improve these symptoms, making quality of life an essential marker of success. Patient satisfaction was similar between the groups but no validated questionnaires were used [20, 23, 24]. The literature on patient satisfaction following haemorrhoidal treatment is scarce. Brown et al. found in a study comparing RBL with HAL found that patient satisfaction after RBL did not differ from HAL in the long term [31].

Murie et al. reported 32 lost days of work after haemorrhoidectomy compared to 3 days after RBL [22]. Time until return to work and normal activities after haemorrhoidectomy has been reported to vary between 9 and 54 days [32]. This wide range can be due to the number of (one-, two-, three-) piles operated or the policy regarding postoperative pain management.

There are numerous studies on treatment of haemorrhoids with various techniques. This illustrates a lack of consensus about when to apply which technique for which 


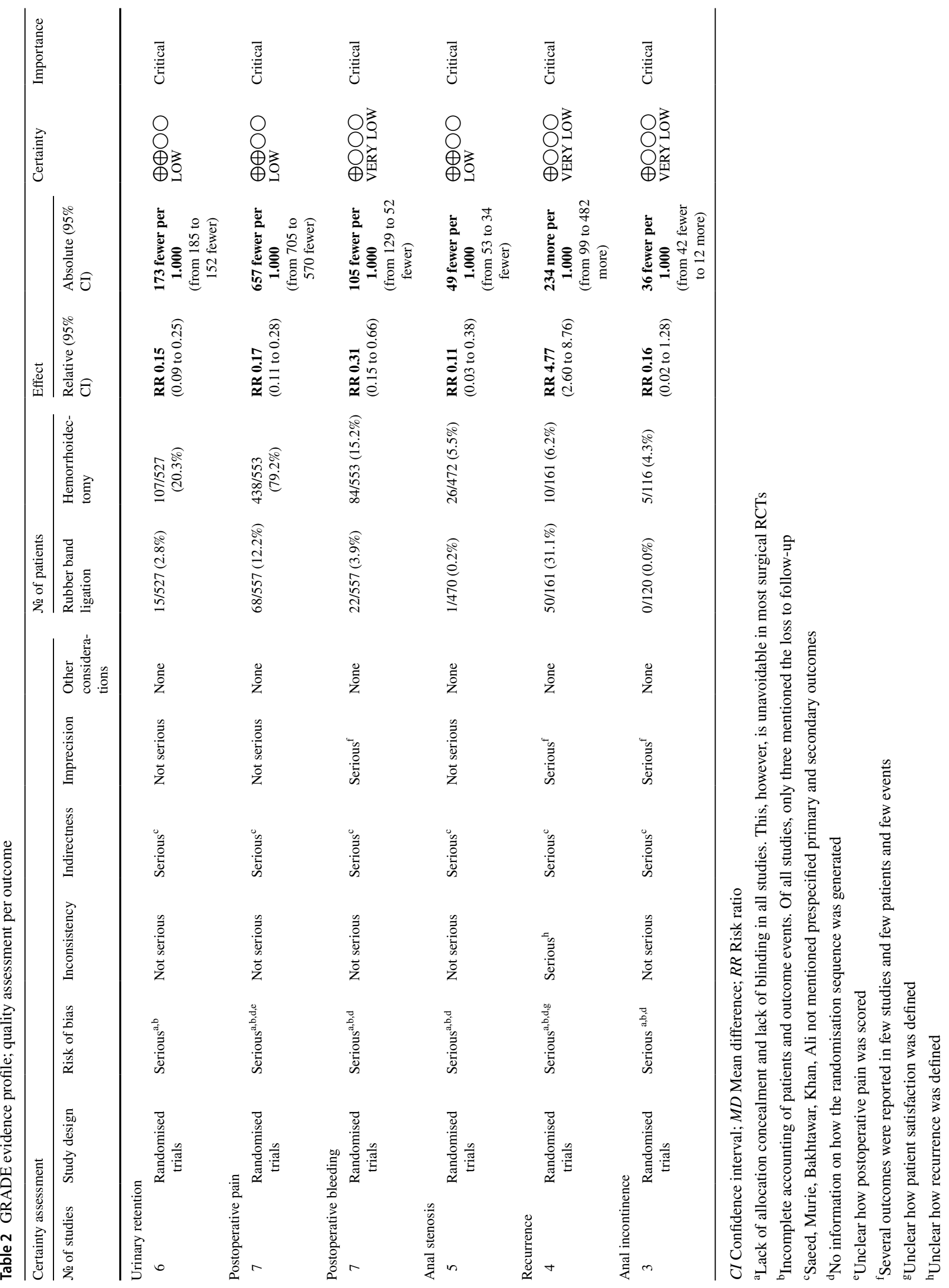




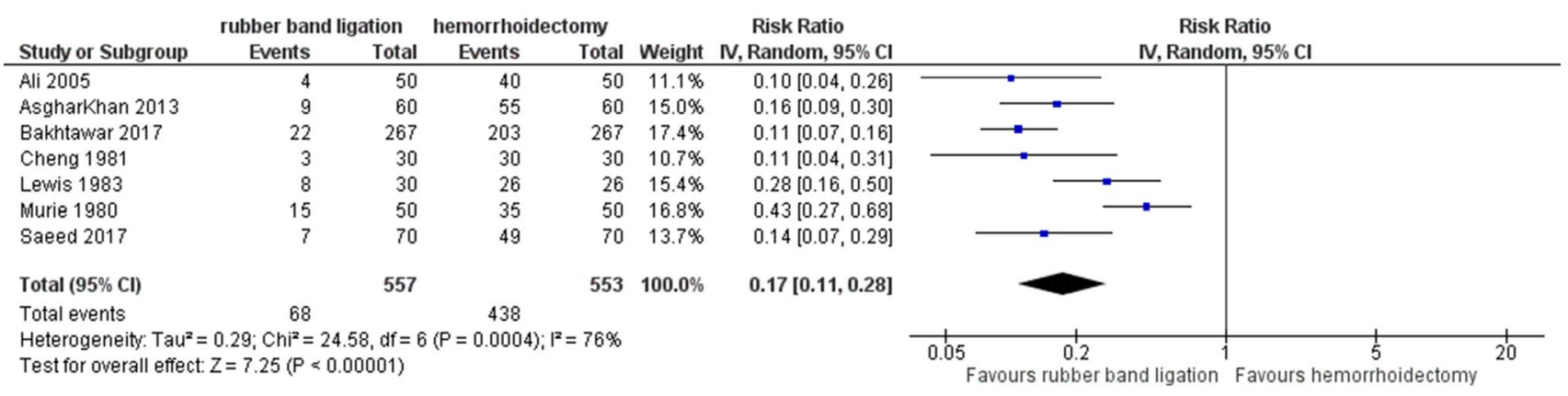

Fig. 4 Postoperative pain. Relative risk values are shown with 95\% confidence intervals

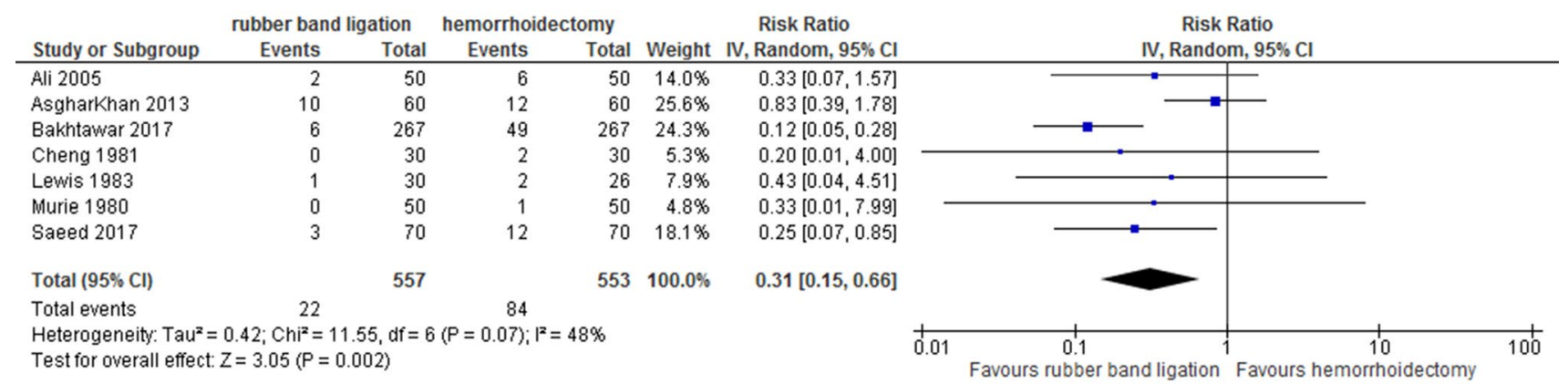

Fig. 5 Postoperative bleeding. Relative risk values are shown with $95 \%$ confidence intervals

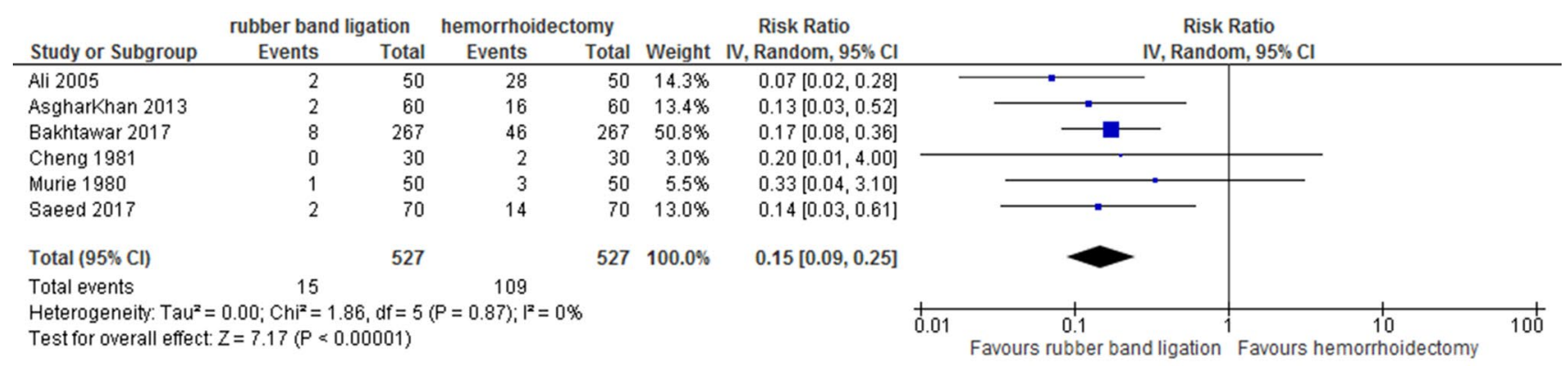

Fig. 6 Urinary retention. Relative risk values are shown with 95\% confidence intervals

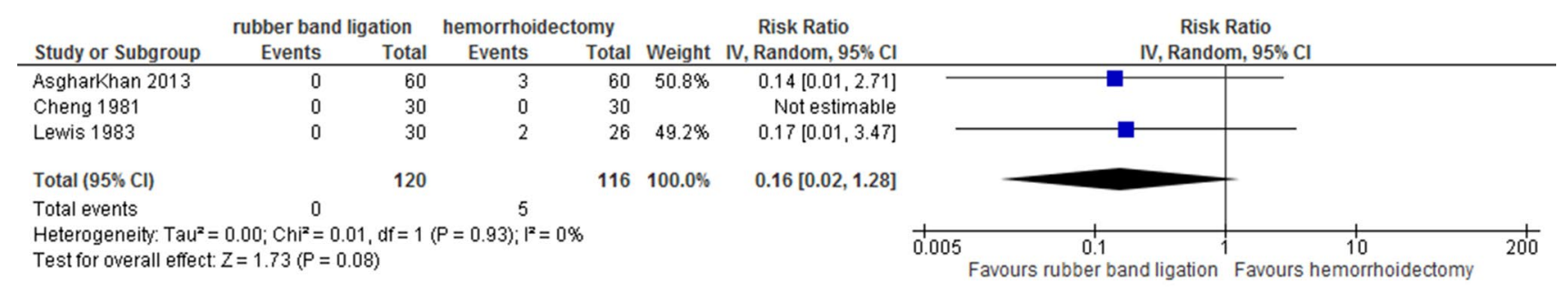

Fig. 7 Anal incontinence. Relative risk values are shown with 95\% confidence intervals 


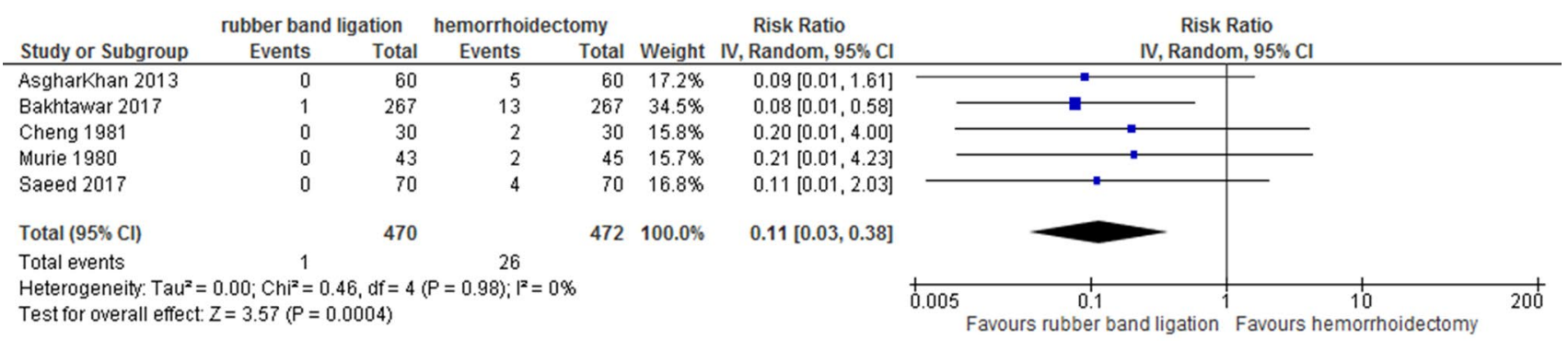

Fig. 8 Anal stenosis. Relative risk values are shown with 95\% confidence intervals

symptoms. Treatment for a benign disease like haemorrhoids has to be safe and should be aimed at relieving symptoms. More conservative methods like RBL are reserved for grade II (but also III) haemorrhoids and more invasive surgical methods for grade III (but also II). That leaves a grey area in which the choice of treatment is not so evident. The gold standard for conservative methods is RBL and the gold standard for surgical procedures is haemorrhoidectomy [33]. Studies comparing these two methods are scarce and only 1 systematic review comparing 3 trials on this subject has been published [9].

Reliable outcome measurements relate to the definition of haemorrhoids. The choice of treatment is mostly based on gradation of haemorrhoids usually based on Goligher's classification [34]. However, symptoms do not reliably relate to Goligher's classification [35]. Clinical evaluation using only the Goligher scale could cause confusion regarding true symptomatic recurrence or symptom persistence. A more solid definition of failure or recurrence together with a validated score of symptoms is indispensable in evaluating treatment [36].

Van Tol et al. recently analysed outcome measurements used in trials on haemorrhoids [37]. Fifty-nine largely varying outcomes were identified. Based on these, the authors developed four different core areas: symptoms, complications, recurrence and resource use/economical impact. When we consider, these core areas in the analysed trials symptoms are only rarely described. None of the studies used a validated symptom score. Recurrence was reported in four studies and was more common following RBL. Complications (postoperative pain, anal stenosis/incontinence, bleeding and urinary retention) were mentioned in 6 studies. Resource use/economical impact was not addressed in any of the studies.

It is also important to realise that haemorrhoidal disease is currently one of the most common disabilities. The condition often leads to disruption in an individual's personal and working life. Management has considerable cost implications, and therefore, economic consequences. None of the included trials mentions costs. Future studies should focus not only on and patient satisfaction with treatment but also on the economic impact of treatment.

\section{Conclusions}

The results of this review suggest that haemorrhoidectomy offers better symptom control compared with rubber band ligation in patients with grade II-III haemorrhoids, but is accompanied by more postoperative pain and complications. The main conclusion, however, must be that the studies analysed are of poor quality, and therefore, no advice about treatment protocol can be given. Good quality trials with an emphasis on economic and patient-related outcomes are needed. A multicentre randomised trial comparing RBL with haemorrhoidectomy has recently been initiated in the Netherlands.

\section{Compliance with ethical standards}

Conflict of interest The authors declare that they have no conflict of interest.

Open Access This article is licensed under a Creative Commons Attribution 4.0 International License, which permits use, sharing, adaptation, distribution and reproduction in any medium or format, as long as you give appropriate credit to the original author(s) and the source, provide a link to the Creative Commons licence, and indicate if changes were made. The images or other third party material in this article are included in the article's Creative Commons licence, unless indicated otherwise in a credit line to the material. If material is not included in the article's Creative Commons licence and your intended use is not permitted by statutory regulation or exceeds the permitted use, you will need to obtain permission directly from the copyright holder. To view a copy of this licence, visit http://creativecommons.org/licenses/by/4.0/. 


\section{References}

1. NIVEL, 'Incidence and prevalence rates of Haemorrhoids in Dutch general practice classified by sex in 2017 (per 1000 patient years)'. Retrieved October 2020 [Online]. Available: https://www. nivel.nl/nl/nivel-zorgregistraties-eerste-lijn/jaarcijfers-aandoening en-huisartsenregistraties.

2. Riss $\mathbf{S}$ et al (2012) The prevalence of hemorrhoids in adults. Int $\mathbf{J}$ Colorectal Dis 27(2):215-220

3. Watson AJM et al (2016) Comparison of stapled haemorrhoidopexy with traditional excisional surgery for haemorrhoidal disease (eTHoS): a pragmatic, multicentre, randomised controlled trial. Lancet 388(10058):2375-2385

4. Simillis C, Thoukididou SN, Slesser AAP, Rasheed S, Tan E, Tekkis PP (2015) Systematic review and network meta-analysis comparing clinical outcomes and effectiveness of surgical treatments for haemorrhoids. Br J Surg 102(13):1603-1618

5. Davis BR, Lee-Kong SA, Migaly J, Feingold DL, Steele SR (2018) The American Society of colon and rectal surgeons clinical practice guidelines for the management of hemorrhoids. Dis Colon Rectum 61(3):284-292

6. van Tol N, Kleijnen RR, Watson J, Jongen AJM, Altomare J, Qvist DF, Higuero SO, Muris T, Breukink J (2019) 'European Society of ColoProctology (ESCP) Guideline for Haemorrhoidal Disease', What do we know so far? CardioVas Interv Radiol 2:1-11

7. van Tol RR, Bruijnen MPA, Melenhorst J, van Kuijk SMJ, Stassen LPS, Breukink SO (2018) A national evaluation of the management practices of hemorrhoidal disease in the Netherlands. Int $\mathbf{J}$ Colorectal Dis 33(5):577-588

8. Altomare DF et al (2018) Surgical management of haemorrhoids: an Italian survey of over 32000 patients over 17 years. Color Dis 20(12):1117-1124

9. Shanmugam V, Thaha MA, Rabindranath KS, Campbell KL, Steele RJC, Loudon MA (2005) Systematic review of randomized trials comparing rubber band ligation with excisional haemorrhoidectomy. Br J Surg 92(12):1481-1487

10. Brown SR, Watson A (2016) Comments to "Rubber band ligation versus excisional haemorrhoidectomy for haemorrhoids." Tech Coloproctol 20(9):659-661

11. McKenzie L et al (2010) 'Economic evaluation of the treatment of grade II haemorrhoids: a comparison of stapled haemorrhoidopexy and rubber band ligation. Colorectal Dis 12(6):587-593

12. Brown SR et al (2016) Haemorrhoidal artery ligation versus rubber band ligation for the management of symptomatic second-degree and third-degree haemorrhoids (HubBLe): a multicentre, open-label, randomised controlled trial. Lancet 388(10042):356-364

13. Moher D et al (2016) Preferred reporting items for systematic review and meta-analysis protocols (PRISMA-P) 2015 statement. Rev Esp Nutr Hum Diet 20(2):148-160

14. 'National Institute for Health Research.' [Online]. Available: https ://www.crd.york.ac.uk/prospero/

15. Higgins JPT et al (2011) The cochrane collaboration's tool for assessing risk of bias in randomised trials'. BMJ 343:7829

16. Guyatt GH et al (2008) GRADE: An emerging consensus on rating quality of evidence and strength of recommendations. BMJ 7:90

17. Ali U, Khan AS (2005) Rubber band ligation versus open haemorrhoidectomy: a study of 100 cases. J Postgrad Med Inst 19(3):317-322

18. Cheng FCY, Shum DWP, Ong GB (1981) The treatment of second degree haemorrhoids by injection, rubber band ligation, maximal anal dilatation, and haemorrhoidectomy: a prospective clinical trial. Aust N Z J Surg 51(5):458-462
19. Bakhtawar A, Khalid MA, Arshad A (2017) Comparison of Milligan - Morgan haemorrhoidectomy vs rubber band ligation in management of haemorrhoids. Med Forum Mon 28(9):83-86

20. Asghar Khan M et al (2013) Short term outcome of rubber band ligation versus open hemorrhoidectomy in terms of postoperative complications. J Med Sci 21(1):19-22

21. Izadpanah A, Hosseini S, Mahjoob M (2010) Comparison of electrotherapy, rubber band ligation and hemorrhoidectomy in the treatment of hemorrhoids: a clinical and manometric study. Middle East J Dig Dis 2(1):9-13

22. Lewis AAM, Rogers HS, Leighton M (1983) Trial of maximal anal dilatation, cryotherapy and elastic band ligation as alternatives to haemorrhoidectomy in the treatment of large prolapsing haemorroids. Br J Surg 70(1):54-56

23. Murie JA, MacKenzie I, Sim AJW (1980) Comparison of rubber band ligation and haemorrhoidectomy for second- and thirddegree haemorrhoids: a prospective clinical trial. Br J Surg 67(11):786-788

24. Saeed MT, Ali Z, Khan SA (2017) Milligan - Morgan (Open) haemorrhoidectomy vs rubber band ligation. Pakistan J Med Heal Sci 11(2):544-548

25. Cocorullo $\mathrm{G}$ et al (2017) The non-surgical management for hemorrhoidal disease. A systematic review. G di Chir 38(1):5-14

26. Watson NFS, Liptrott S, Maxwell-Armstrong CA (2006) A prospective audit of early pain and patient satisfaction following out-patient band ligation of haemorrhoids. Ann R Coll Surg Engl 88(3):275-279

27. Zaheer S, Reilly WT, Pemberton JH, Ilstrup D (1998) Urinary retention after operations for benign anorectal diseases. Dis Colon Rectum 41(6):696-704

28. Toyonaga $\mathrm{T}$ et al (2006) Postoperative urinary retention after surgery for benign anorectal disease: potential risk factors and strategy for prevention. Int J Colorectal Dis 21(7):676-682

29. Kowalik U, Plante MK (2016) Urinary retention in surgical patients. Surg Clin North Am 96(3):453-467

30. Trenti L, Biondo S, Galvez A, Bravo A, Cabrera J, Kreisler E (2017) Distal Doppler-guided transanal hemorrhoidal dearterialization with mucopexy versus conventional hemorrhoidectomy for grade III and IV hemorrhoids: postoperative morbidity and long-term outcomes. Tech Coloproctol 21(5):337-344

31. Brown $\mathrm{S}$ et al (2016) The HubBle trial: Haemorrhoidal artery ligation (HAL) versus rubber band ligation (RBL) for symptomatic second- and third-degree haemorrhoids: a multicentre randomized controlled trial and health-economic evaluation. Health Technol Assess (Rockv) 20(88):1-180

32. Shao WJ, Li GCH, Zhang ZHK, Yang BL, Sun GD, Chen YQ (2008) Systematic review and meta-analysis of randomized controlled trials comparing stapled haemorrhoidopexy with conventional haemorrhoidectomy. Br J Surg 95(2):147-160

33. MacRae HM, McLeod RS (1995) Comparison of hemorrhoidal treatment modalities - A meta-analysis. Dis Colon Rectum 38(7):687-694

34. Goligher JC (1980) 'Surgery of the anus Rectum and colon. Bailliere Tindall 3:924-925

35. Gerjy R, Lindhoff-Larson A, Nyström PO (2008) Grade of prolapse and symptoms of haemorrhoids are poorly correlated: result of a classification algorithm in 270 patients. Color Dis 10(7):694-700

36. Rørvik HD et al (2019) Hemorrhoidal disease symptom score and short health scaleHD: New tools to evaluate symptoms and health-related quality of life in hemorrhoidal disease. Dis Colon Rectum 62(3):333-342

37. van Tol RR et al (2019) European Society of Coloproctology Core Outcome Set for haemorrhoidal disease: an international Delphi study among healthcare professionals. Color Dis 21(5):570-580 
Publisher's Note Springer Nature remains neutral with regard to jurisdictional claims in published maps and institutional affiliations. 